Muro de la Investigación, 2018: 3(1), Enero-Julio, ISSN: 2523-2886

DOI:https://doi.org/10.17162/rmi.v3i1.1110

\title{
Efectividad del programa "soy creativo" para el desarrollo de la inteligencia creativa, en niños del segundo grado del nivel primario, del Liceo y Academia Adventista Diéguez Olaverri, Huehuetenango, Guatemala. 2017
}

\author{
Keili Johana Jiménez Jiménez ${ }^{1 \mathrm{a}}$ y Salomón Vásquez Villanueva² \\ Universidad Peruana Unión, Perú12
}

\section{Recibido: 12 de enero de 2018 Aceptado: 05 de mayo de 2018}

\begin{abstract}
Resumen
El objetivo de este estudio fue determinar en qué medida el programa de intervención psicopedagógica es efectivo para el desarrollo de la inteligencia creativa en los niños de segundo año de primaria del Liceo y Academia Adventista Diéguez Olaverri. El tipo de investigación es pre-experimental, programa de intervención psicopedagógico "Soy Creativo", para determinar los efectos sobre la variable inteligencia creativa, en una muestra de 20 niños de $2^{\circ}$ de primaria del Liceo y Academia Adventista Diéguez Olaverri en la cuidad de Huehuetenango, Guatemala. Para la presente investigación se construyeron dos instrumentos de acuerdo con los propósitos de la misma: una fue respondida por los niños participantes y otra por la maestra investigadora, ambas realizadas antes y después del desarrollo del programa. Los datos recabados fueron procesados mediante el programa SPSS 22.0, la hipótesis demostró usando la prueba t de studen para muestras relacionadas. Se concluyó que el programa es efectivo en la mejorar de la inteligencia creativa de los estudiantes, al haber una diferencia significativa entre las pruebas de entrada y salida $(\mathrm{p}<$ $0.005)$.
\end{abstract}

Palabras clave: inteligencia creativa, programa de intervención psicopedagógica, creatividad.

\section{Effectiveness of the "I am creative" program for the development of creative intelligence, in children of the second grade of the primary level, of the Liceo and Diéguez Adventist Academy in Olaverri, Huehuetenango, Guatemala. 2017}

\footnotetext{
${ }^{\text {a }}$ Correspondencia al autor

E-mail: keilijimenez@upeu.edu.pe
} 


\begin{abstract}
The objective of this study was to determine the effectiveness of the program for psychopedagogical intervention in the development of creative intelligence among children from the second year of elementary school of the Liceo and Academia Adventista Diéguez Olaverri. The type of research is pre-experimental, psycho-pedagogical intervention program "Soy Creativo", to determine the effects on the variable creative intelligence, in a sample of 20 children from 2nd grade of the Liceo and Diéguez Olaverri Adventist Academy in the city of Huehuetenango, Guatemala. For this study, two instruments were constructed according to the purposes of the research: one was answered by the participating children and the other by the research teacher, both carried out before and after the development of the program. The data collected were processed through the SPSS 22.0 program, the hypothesis was demonstrated using Student's t test for related samples. It was concluded that the program is effective in improving students' creative intelligence, as there is a significant difference between the entrance and exit tests $(\mathrm{p}<0.005)$.
\end{abstract}

Key-words: creative intelligence, psychopedagogical intervention program, creativity

\title{
Introducción
}

En el estudio se ha trabajado dos variables: la variable independiente denominada Programa de intervención psicopedagógico "Soy creativo". La variable dependiente: inteligencia creativa, cuyas dimensiones fueron cuatro: personalidad creativa, proceso creativo, producto creativo y contexto/ambiente creativo. Para consolidar el estudio se trabajaron las bases teóricas conformadas por el marco histórico, el marco filosófico, el marco teórico y el marco conceptual.

Entre otros antecedentes, se analizó el estudio doctoral realizado por Soto (2013), cuyo programa fue elaborado para el Desarrollo del Pensamiento Divergente Infantil (DEPDI), atendiendo el objetivo general de permitir un incremento significativo en el pensamiento creativo de los estudiantes de segundo ciclo de educación inicial, en el ámbito de un estudio experimental, los investigadores manipularon intencionalmente mediante una acción, en una escuela infantil de Madrid, comunidad de Gatafe, durante un curso lectivo (octubre-junio), en una muestra de 57 niños entre 3 y 6 años, organizados en 3 grupos clasificados por edades: el primer subgrupo: 14 niños de 3 años; el segundo: 26 niños de 4 años y el tercer subgrupo: 17 niños de 5 años. A los niños se les aplicó el programa; a los padres y maestros se les aplicó un pretest y un postest, para confirmar las hipotesis: finalizado el programa, los niños tendrían un cambio notable en la creatividad y el desarrollo del pensamiento divergente. Según los resultados, a mayor edad hubo mayor significancia, permite concluir que la experiencia previa le suma mayores habilidades en el 
desarrollo del pensamiento divergente. Las conclusiones finales exponen que los niños y niñas quienes recibieron las sesiones de aprendizaje del programa, tuvieron un notable desempeño para el desarrollo del pensamiento divergente, determinando que se cumplieron los objetivos propuestos por la autora.

El objetivo de este artículo fue determinar en qué medida el programa de intervención psicopedagógica "Soy creativo" es efectivo para el desarrollo de la inteligencia creativa en los niños de segundo año de primaria del Liceo y Academia Adventista Diéguez Olaverri.

\section{Referencias teóricas}

Enfoques teóricos de la creatividad

El tema de la creatividad se ha analizado desde diferentes disciplinas: la psicología, la sociología, la epistemología, el psicoanálisis, la ciencia el arte, la cultura, etc. Cada una de estas áreas del conocimiento tiene un enfoque, desde el cual sus autores definen este constructo y presentan sus modelos teóricos.

Teorías implícitas

Las teorías basadas sobre la personalidad creativa están enfocadas en el individuo y todas las capacidades que lo hacen un ser creador. Según Wegner y Vallacher (1977, citados por Prado, 2004), "las teorías implícitas son aquellas teorías hechas por el hombre de la calle con el fin de explicar, controlar y redecir futuros eventos". A continuación, se presentan las principales teorías implícitas de la creatividad.

Teoría de la búsqueda de sí mismo

La base teórica de este enfoque está dirigida a la personalidad creativa que se refleja en su creación, según Rodríguez (citado por Henao \& Berrío, 2014) existe una reciprocidad entre el producto final y su creador, ya que "las creaciones se convierten en una prolongación de las personas a través de tiempo y el espacio" ya que los representa, aunque se encuentre ausente por distancia o muerte. Según Obradors (2007), la teoría de la búsqueda de uno mismo usa la expresión artística o creativa: camino para llegar al conocimiento profundo de su ser interior. El perfil de tal artista tiene características intelectuales, pero de gran introspección que lo aleja de mundo externo completamente. Convirtiéndose la obra de arte en obsesión y el espejo de sí mismo. 
Entonces para la teoría de la búsqueda de sí mismo, la creatividad es un proceso en que la persona creativa hace una exploración interior profunda de su ser y al final del proceso resulta una obra o producto creativo que refleja el carácter mismo del artista.

Teoría de la expresión emocional

La teoría de la expresión emocional se refiere como su nombre lo indica, a los estados emocionales que impulsan a la persona al hacer creativo. Para Obradodors (2007), los conflictos internos y los estados emocionales son la fuente principal de inspiración para el artista, esta teoría coincide con la Freudiana en que la creatividad es el resultado directo de los problemas emocionales del ser, en donde existe lucha entre el consciente y el inconsciente.

De acuerdo con Romo (citado por Fuentes \& Torbay, 2004) a través del arte creativo, el artista hace una catarsis que le permite aflorar todos sus sentimientos y todas las emociones son de inspiración para la creación de sus obras más hermosas. Para llegar a esta experiencia, quien crea debe buscar nuevas prácticas que le permitan destacar sus más profundos sentimientos, además se asocia al genio creativo con rasgos de personalidad específicas: tendencia al consumo de estupefacientes y una introversión extrema con cuadros extrovertidos exagerados. En pocas palabras, se puede decir que la base de esta teoría son las emociones como fuente de inspiración creativa.

Teoría de comunicación

En esta teoría, el quehacer creativo se define como el medio a través del cual se busca producir sentimientos, por eso el artista que lograba impactar con sus creaciones artísticas se les consideraba inspirados por el espíritu de dios (Obradors, 2007, p. 44, 45)

Según Romo (1997), la iglesia católica tenía como mito que las obras de arte que lograban transmitir fervor y sublimidad eran terminadas por la virgen María. A diferencia de la teoría de expresión emocional, la creación no se agota al finalizar su ejecución, sino que su pico más alto es cuando el producto final es expuesto para deleite de los presentes.

Entonces se puede decir que, en esta teoría, la creatividad se destaca cuando el producto final es puesto a disposición del público, quien le da un alto valor si la obra creativa logra conmoverle.

Teoría dotes especiales innatas

Los autores más destacados de esta teoría son Kris y Kurs, quienes en 1934 escriben un libro titulado: La leyenda del artista. En donde se narra la historia del artista humilde, quien desde 
la infancia destaca por sus dotes artísticas innatos, su lucha se da por expresarse y para que surja su talento pese al ambiente adverso. La historia termina con un final feliz de reconocimiento del artista y ascenso social.

Según Romo (citado por Henao \& Berrío, 2014), la teoría de dotes especiales se consolida en el renacimiento, se destaca el artista como poseedor de la inspiración, la cual es la visión interior de quien está poseído por la divinidad. Es decir, su genio es propio en él desde su nacimiento y para los demás mortales es imposible llegar hasta su nivel. La sentencia se resume así: "el artista nace, no se hace".

Por su parte, Velasco (2007) concuerda con Romo al expresar que esta teoría implícita representa una de las principales creencias de la mitología artística, la cual cree que el genio creativo tiene capacidades innatas muy sobresalientes al resto de los mortales, se cree además en el campo psicológico que dichos rasgos pueden ser hereditarios.

Teorías referidas al proceso creativo

El proceso creativo según Gordon (citado por Romero-infante \& Moré-Jaramillo, 2013) radica en la actividad mental en situaciones de definición problemas o solución de problema, cuyo producto son las invenciones artísticas o técnicas, acentuando así tanto la formulación como la solución de problemas como partes proceso creativo.

El proceso es parte fundamental en la actividad creadora, porque de él se espera como resultado el producto creativo, el cual es un objeto externo, para ser valorado creativo o no, según su utilidad, apariencia y novedad. Para que el proceso se dé completo se deben tener en cuenta viarias fases o etapas. Existen diferentes clasificaciones. La más popular es la propuesta por Graham Wallas en su libro "El arte del pensamiento" (1926).

Según Graham Wallas (1926, citado por Sastre-Riba \& Pascual-Sufrate, 2013) las etapas del proceso creativo son:

Preparación, en esta primera etapa se da la concientización o definición del problema o necesidad, también es conocida por la etapa de sensibilización en la que principalmente destaca la preocupación de mejorar la realidad.

Incubación, esta corresponde al tiempo casi indefinido que conlleva una documentación o investigación. Durante este periodo, la persona trabaja y reflexiona de modo inconsciente en el problema. 
Iluminación, si la fase de incubación tiene éxito, la persona experimenta una súbita iluminación que corresponde a una solución novedosa del problema inicial. También se puede comparar con el "Eureka" de Arquímedes, que surge después de un cúmulo de envolvente de preguntas y respuestas.

Verificación, en esta etapa final se realiza la selección de todos procedimientos que satisfacen la necesidad inicial, para posteriormente preparar la plataforma que permite la solución del problema.

\section{Metodología}

Esta investigación se enmarca en el tipo pre-experimental, ya que a partir de la manipulación de la variable independiente se pretende observar los efectos y su relación con la variable dependiente (Hernández, Fernández, \& Baptista, 2010).

El diseño de preprueba y posprueba con un solo grupo, es decir, a la muestra se le aplicará una prueba de entrada seguida del estímulo (el programa de intervención), finalmente se suministra la prueba de salida que determinará si hubo cambios significativos en el grupo, después de la manipulación de la variable independiente, este diseño se diagrama de la

Siguiente manera (Hernández et al., 2010):

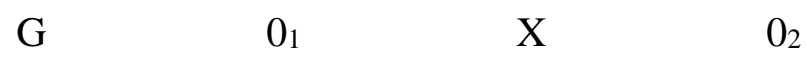

Donde:

$\mathrm{G}=$ Grupo

$01=$ Prueba de entrada

$\mathrm{X}=$ Programa de intervención Psicopedagógica "Soy Creativo"

02 = Prueba de salida

La población y muestra de la investigación está compuesta por 20 estudiantes de Educación Primaria del $2^{\circ}$ grado del Liceo y Academia Adventista Diéguez Olaverri. De este grupo de 20 estudiantes, 12 son niños y 8 son niñas, tienen edades comprendidas entre los 7 y 10 años. Además, se contó con la participación de una niña de 13 años que tiene necesidades educativas especiales, por los cual se aplicó el criterio de inclusión y exclusión.

El proceso de ejecución del presente trabajo de investigación se inició con la coordinación y autorización de las autoridades educativas de la Institución Educativa en mención, agotando la 
parte legal, luego se aplicó la propuesta del trabajo de investigación; es decir, la ejecución del programa de intervención psicopedagógica "Soy Creativo". Finalmente se recopilaron los datos a través de las pruebas: de entrada y salida. Tal procesamiento de datos y pruebas estadísticas se realizó utilizando el programa SPSS 22.0.

\section{Instrumentos utilizados}

Para esta investigación se usaron dos instrumentos principales.

Cuestionarios

Consiste en veinte (20) ítems referidos a la inteligencia creativa la cual se aplica como prueba de entrada y de salida que se aplicaron a la muestra seleccionada. Este cuestionario abarca las cuatro (4) dimensiones de la presente investigación: la personalidad creativa, el proceso creativo, el producto creativo y el contexto/ambiente creativo.

Prueba para niños

Consiste en cuatro (4) ítems, se evalúa el Programa de intervención psicopedagógica "Soy creativo". El programa de intervención psicopedagógico que pretende mejorar la inteligencia creativa en los sujetos, objeto de observación.

Técnicas, procesamiento y análisis de los datos obtenidos

Para ejecutar el tratamiento de las evaluaciones hechas a los estudiantes, se realizó el siguiente proceso: La aplicación de la prueba de entrada a los 21 estudiantes del segundo grado $\left(2^{\circ}\right)$ de primaria del Liceo y Academia Adventista Diéguez Olaverri. La calificación de la prueba de entrada a los 21 estudiantes del segundo grado $\left(2^{\circ}\right)$. Desarrollo del programa de intervención psicopedagógica "soy creativo", para desarrollar la inteligencia creativa a los 21 estudiantes de la muestra seleccionada. La aplicación de la prueba de salida a los 20 estudiantes del segundo grado $\left(2^{\circ}\right)$ de primaria. La calificación de la prueba de salida a los 20 estudiantes del segundo grado $\left(2^{\circ}\right)$ de primaria. Los datos son registrados, utilizando el paquete estadístico SPSS 22.0, siguiendo los parámetros estadísticos respectivos, determinando la media, mediana, varianza, entre otros. Los datos son presentados en tablas estadísticas. La interpretación de los datos es presentada según corresponde a cada tabla estadística presentada.

\section{Resultados}

Resultados 1

Tabla 01 
Prueba de T de student por la diferencia de las pruebas de entrada y salida

\begin{tabular}{|c|c|c|c|c|c|c|c|c|}
\hline & \multicolumn{5}{|c|}{ Diferencias emparejadas } & \multirow{3}{*}{$\mathrm{t}$} & \multirow{3}{*}{ gl } & \multirow{3}{*}{$\begin{array}{c}\text { Sig. } \\
\text { (bilateral) }\end{array}$} \\
\hline & \multirow{2}{*}{ Media } & \multirow{2}{*}{$\begin{array}{l}\text { Desviación } \\
\text { estándar }\end{array}$} & \multirow{2}{*}{$\begin{array}{l}\text { Media de } \\
\text { error } \\
\text { estándar }\end{array}$} & \multicolumn{2}{|c|}{$\begin{array}{c}95 \% \text { de intervalo de } \\
\text { confianza de la diferencia }\end{array}$} & & & \\
\hline & & & & Inferior & Superior & & & \\
\hline $\begin{array}{l}\text { Pos test } 1 \\
\text { - Pre test } \\
1\end{array}$ & 0.650 & 0.671 & 0.150 & 0.336 & 0.964 & 4.333 & 19 & .000 \\
\hline
\end{tabular}

\section{Fuente propia}

En la tabla 01, se observa una gran diferencia de la media del pos test en relación con el pre test. La diferencia de las medias es de 0.650 puntos. La diferencia mediante $\mathrm{T}$ de student es de 4.333. Se rechaza la hipótesis nula porque el valor de $\mathrm{p}=0.000$ que es mayor que alfa $=0.05$.

Se concluye que existe una diferencia significativa entre los resultados del pre test de la variable desarrollo de la personalidad creativa y el pos test de la misma, lo que significa que el programa que se aplicó fue efectivo y los resultados mejoraron de manera considerable.

Tabla 02

Prueba de T de student por la diferencia de las pruebas de entrada y salida

\begin{tabular}{|c|c|c|c|c|c|c|c|c|}
\hline & \multicolumn{5}{|c|}{ Diferencias emparejadas } & \multirow{3}{*}{$\mathrm{T}$} & \multirow{3}{*}{$\mathrm{gl}$} & \multirow{3}{*}{$\begin{array}{c}\text { Sig. } \\
\text { (bilateral) }\end{array}$} \\
\hline & \multirow{2}{*}{ Media } & \multirow{2}{*}{$\begin{array}{l}\text { Desviación } \\
\text { estándar }\end{array}$} & \multirow{2}{*}{$\begin{array}{l}\text { Media de } \\
\text { error } \\
\text { estándar }\end{array}$} & \multicolumn{2}{|c|}{$\begin{array}{l}95 \% \text { de intervalo de } \\
\text { confianza de la diferencia }\end{array}$} & & & \\
\hline & & & & Inferior & Superior & & & \\
\hline $\begin{array}{l}\text { Pos test } \\
3 \text { - Pre } \\
\text { test } 3\end{array}$ & 1.450 & 1.146 & 0.256 & 0.914 & 1.986 & 5.659 & 19 & .000 \\
\hline
\end{tabular}

Fuente propia

En la tabla 02, se observa una gran diferencia de la media del pos test con relación al pre test. La diferencia de las medias es de 1.450 puntos. La diferencia mediante $\mathrm{T}$ de student es de 5.659. Se rechaza la hipótesis nula porque el valor de $\mathrm{p}=0.000$ es menor que alfa $=0.05$.

Se concluye que existe una diferencia significativa entre los resultados del pre test de la dimensión del desarrollo del desarrollo del producto creativo y el pos test de la misma, lo que significa que el programa que se aplicó fue efectivo y los resultados mejoraron de manera considerable.

Tabla 03

Prueba de T de student por la diferencia de las pruebas de entrada y salida 


\begin{tabular}{|c|c|c|c|c|c|c|c|c|}
\hline & \multicolumn{5}{|c|}{ Diferencias emparejadas } & \multirow{3}{*}{$\mathrm{t}$} & \multirow{3}{*}{ gl } & \multirow{3}{*}{$\begin{array}{c}\text { Sig. } \\
\text { (bilateral) }\end{array}$} \\
\hline & \multirow[t]{2}{*}{ Media } & \multirow{2}{*}{$\begin{array}{l}\text { Desviación } \\
\text { estándar }\end{array}$} & \multirow{2}{*}{$\begin{array}{l}\text { Media de } \\
\text { error } \\
\text { estándar }\end{array}$} & \multicolumn{2}{|c|}{$\begin{array}{c}95 \% \text { de intervalo de } \\
\text { confianza de la } \\
\text { diferencia }\end{array}$} & & & \\
\hline & & & & Inferior & Superior & & & \\
\hline $\begin{array}{l}\text { Pos test } 4 \\
\text { - Pre test } 4\end{array}$ & 1.100 & 0.912 & 0.204 & 0.673 & 1.527 & 5.395 & 19 & .000 \\
\hline
\end{tabular}

\section{Fuente propia}

En la tabla 03, se observa una gran diferencia de la media del pos test con relación al pre test. La diferencia de las medias es de 1.100 puntos. La diferencia mediante $\mathrm{T}$ de student es de 5.395. Se rechaza la hipótesis nula, porque el valor de $\mathrm{p}=0.000$ es menor que alfa=0.036.

Se concluye que existe una diferencia significativa entre los resultados del pre test de la dimensión desarrollo del ambiente creativo y el pos test de la misma, lo que significa que el programa que se aplicó fue efectivo y los resultados mejoraron de manera considerable.

Tabla 04

Prueba de T de student por la diferencia de las pruebas de entrada y salida

\begin{tabular}{|c|c|c|c|c|c|c|c|c|}
\hline & \multicolumn{5}{|c|}{ Diferencias emparejadas } & \multirow{3}{*}{$\mathrm{t}$} & \multirow{3}{*}{$\mathrm{gl}$} & \multirow{3}{*}{$\begin{array}{c}\text { Sig. } \\
\text { (bilateral) }\end{array}$} \\
\hline & \multirow[t]{2}{*}{ Media } & \multirow{2}{*}{$\begin{array}{l}\text { Desviación } \\
\text { estándar }\end{array}$} & \multirow{2}{*}{$\begin{array}{l}\text { Media de } \\
\text { error } \\
\text { estándar }\end{array}$} & \multicolumn{2}{|c|}{$\begin{array}{c}95 \% \text { de intervalo de } \\
\text { confianza de la } \\
\text { diferencia } \\
\end{array}$} & & & \\
\hline & & & & Inferior & Superior & & & \\
\hline $\begin{array}{l}\text { Pos test } \\
\text { va - Pre } \\
\text { test va }\end{array}$ & 3.550 & 2.064 & 0.462 & 2.584 & 4.516 & 7.692 & 19 & .000 \\
\hline
\end{tabular}

\section{Fuente propia}

En la tabla 04, se observa una gran diferencia de la media del pos test con relación al pre test. La diferencia de las medias es de 3.550 puntos. La diferencia mediante $\mathrm{T}$ de student es de 7.692. Se rechaza la hipótesis nula porque el valor de $\mathrm{p}=0.000$ que es mayor que alfa=0.05.

Se concluye que existe una diferencia significativa entre los resultados del pre test de la dimensión inteligencia creativa y el pos test de la misma, lo que significa que el programa que se aplicó fue significativamente efectivo y los resultados mejoraron de manera considerable.

\section{Conclusión}


El programa de intervención psicopedagógica, de acuerdo con el estudio y sus resultados respectivos, fue efectivo para el desarrollo de la inteligencia creativa en los niños de segundo año de primaria del Liceo y Academia Adventista Diéguez Olaverri.

El programa de intervención psicopedagógica, en forma significativa, fue efectivo para el desarrollo de la personalidad creativa en los niños de segundo año de primaria del Liceo y Academia Adventista Diéguez Olaverri.

Por otro lado, el programa de intervención psicopedagógica fue efectivo, en forma significativa, para el desarrollo del proceso creativo.

En forma significativa, el programa de intervención psicopedagógica fue efectivo para el desarrollo del producto creativo.

También el programa de intervención psicopedagógica fue efectivo, en forma significativa, para el desarrollo del Contexto/ambiente creativo.

\section{Referencias}

Fuentes, C., \& Torbay, Á. (2004). Desarrollar la creatividad desde los contextos educativos: un marco de reflexión sobre la mejora socio-personal. Revista Electrónica Iberoamericana sobre Calidad, Eficacia y Cambio en Educación, 2(1), 1-14. Recuperado a partir de https://revistas.uam.es/index.php/reice/article/view/5541

Henao, B., \& Berrío, L. (2014). Relación entre teorías implicitas de creatividad de los docentes universitarios y los estudiantes de licenciatura en educación [Tesis de maestría]. Universidad de Antioquia. Recuperado a partir de http://ayura.udea.edu.co:8080/jspui/handle/123456789/627

Hernández, R., Fernández, C., \& Baptista, M. del P. (2010). Metodología de la investigación. (J. Mares, Ed.), Metodología de la investigación (5a. ed.). México, D.F: McGRAWHILL/INTERAMERICANA EDITORES, S.A. DE C.V. https://doi.org/- ISBN 978-92-7532913-9

Obradors, M. (2007). Creatividad y Generación de Ideas: Estudio de la práctica creativa en cine y publicidad. Barcelona: Aldea Global. 
Prado, R. (2004). Creatividad y sobredotación Diagnóstico e Intervención Psicopedagógica (Tesis doctoral). Universidad de Málaga. Recuperado a partir de http://www.biblioteca.uma.es/bbldoc/tesisuma/16801118.pdf

Romero-infante, J. A., \& Moré-jaramillo, R. A. (2013). Sistema de solución creativa para problemas recurrentes - Itacone. Ingeniería y Competitividad, 15(1), 21-35. Recuperado a partir de http://www.redalyc.org/html/2913/291329165002/

Romo, M. (1997). Psicología de la creatividad. Barcelona: Paidós Iberica, S.A.

Sastre-Riba, S., \& Pascual-Sufrate, M. T. (2013). Alta capacidad intelectual, resolución de problemas y creatividad. Revista de neurología, 56(1), 67-76. Recuperado a partir de http://www.ncbi.nlm.nih.gov/pubmed/23446726

Soto, V. (2013). Diseño y aplicación de un programa de creatividad para el desarrollo del pensamiento divergente en Segundo Ciclo de Educación Infantil [Tesis doctoral]. Universidad Complutense de Madrid. Recuperado a partir de http://eprints.ucm.es/22396/1/T34662.pdf Velasco, P. (2007). Psicología y creatividad: Una revisión historica (Desde los autorretratos de los genios del siglo XIX hasta las teorías implícitas del siglo XX). Caracas: Fondo Editorial de la Facultad de Humanidades y Educación. Universidad Central de Venezuela. 